\title{
The Clinical and Biomechanical Performance of All-Suture Anchors: A Systematic Review
}

\author{
Selim Ergün, M.D., Umut Akgün, M.D., F. Alan Barber, M.D., and Mustafa Karahan, M.D.
}

Purpose: This systematic review aimed to clarify the relative strengths and weaknesses of the all-suture anchors (ASAs) in both clinical and experimental studies. Our hypothesis was that there would be similar clinical and experimental data for ASAs regarding the biomechanical properties, clinical outcomes and complication rates. Methods: A systematic review of MEDLINE and Embase databases was performed. The inclusion criteria for clinical studies were both retrospective or prospective study design and minimum l-year follow-up; for biomechanical studies, the inclusion criteria were performance on either cadaver and animal bones or synthetic surfaces. Studies were excluded if the studies were not in English or if they were review articles, commentaries, letters, case reports, or technical notes. The risk of bias assessment was done using the Methodological Index for Non-randomized Studies (MINORS) tool. Results: We included 13 experimental and 3 clinical studies. The least displacement under cyclic loading was recorded with Q-Fix. Failure mode was mostly by suture breaking for the Q-Fix, whereas anchor pullout was the most common for the others. Cadaver humerus' greater tuberosity seemed to be less durable for the ASAs. Tests on cadaver glenoid showed similar biomechanical properties when compared to a control anchor. Studies investigating clinical and radiologic findings were very few, and only 3 case series were included in this review. Clinical findings of patients treated with ASAs for instability and rotator cuff repair showed satisfactory results and little increase in the complication rate (retear or revision surgery because of loose anchor). Conclusions: ASAs have similar or better biomechanical properties compared to regular anchors. Lowprofile design seems to be an important advantage. Case series can not distinguish between the possible clinical benefits and/or risks. Clinical Relevance: ASAs have similar biomechanical properties when compared with other types of anchors. Their strength and performance vary with anatomic location, which may influence clinical success.

$\mathbf{F}$ or the purpose of reattachment of tendons and other soft tissues to bone, suture anchors are widely used with minimally invasive techniques, especially in the upper extremity. These devices have been improved over time, with the ability to accommodate multiple sutures, the use of biodegradable and biocomposite materials and

From the Department of Orthopedics and Traumatology, School of Medicine, Acibadem Mehmet Ali Aydinlar University, Istanbul, Turkey (S.E., U.A. M.K.; Plano Orthopedic and Sports Medicine Center, Plano, Texas, U.S.A. (F.A.B.).

The authors report no conflicts of interest in the authorship and publication of this article. Full ICMJE author disclosure forms are available for this article online, as supplementary material.

Received October 2, 2019; accepted February 18, 2020.

Address correspondence to Selim Ergün, M.D., Acibadem Kozyatagr Hastanesi, Okur sokak, no: 24, 34734, Kozyatağı, Istanbul. E-mail: drselimergun@gmail.com

(C) 2020 THE AUTHORS. Published by Elsevier Inc. on behalf of the Arthroscopy Association of North America. This is an open access article under the CC BY-NC-ND license (http://creativecommons.org/licenses/by-nc-nd/4.0/). 2666-061X/191167

https://doi.org/10.1016/j.asmr.2020.02.007 knotless designs. Suture anchors made completely of suture material were developed in the past decade. These all-suture anchors (ASAs) are based on ultrahigh molecular-weight polyethylene-containing sutures. The anchor portion of the device typically consists of a sleeve or tape, also made from suture material, through which the ultrahigh molecular-weight polyethylene-containing suture is woven. When the ASA is inserted into bone and the suture limbs are pulled, the sleeve or tape is cinched up to compress against the overlying cortical bone creating a "ball," which serves as the anchor. Potential benefits include decreased bone damage. They are radiolucent and nondegradable. ASAs used for the glenoid are smaller and usually have 1 or, at most, 2 sutures, whereas those used for rotator cuff tendon repairs are larger and are either double- or triple-loaded. ${ }^{1,2}$

Biomechanical testing reveals the failure mode and strength of ASAs and shows that they have sufficient strength for soft tissue-to-bone healing in the majority of clinical settings. ${ }^{3}$ However, most biomechanical studies are in vitro and done at time 0 , and only a few in vivo animal studies, which may not reflect the 
human clinical response, are available. ${ }^{3-15}$ A literature search of clinical outcomes and complications related to ASAs discovered relatively few studies. ${ }^{16-18}$

This study sought to address the following questions: (1) Because it is not feasible to obtain ASA biomechanical data clinically, is that high failure strength consistent with the clinical experience?; (2) Is tunnel widening or cyst formation previously reported in animal studies reported clinically? ${ }^{7}$

This systematic review focused on biomechanical test results and clinical outcomes of different sizes and models of ASAs used for soft tissue-to-bone fixation. Our hypothesis was that clinical outcomes and complication rates of ASAs are correlated with their biomechanical test results.

\section{Methods}

This study was performed according to the Preferred Reporting Items for Systematic reviews and Metaanalyses (PRISMA). ${ }^{19}$ MEDLINE and Embase databases were searched for published randomized or nonrandomized controlled studies and prospective or retrospective case series presenting the clinical results of soft-tissue repairs using ASAs and also experimental studies testing the biomechanical properties of ASAs in cadavers and animals and on synthetic surfaces. We independently searched the databases for the key phrases "all-suture anchor" OR "ASA." All articles that were identified using these search terms on February 15,2019 , were then reviewed and discussed among the authors, and a decision whether to include or exclude them was made according to inclusion and exclusion criteria. The inclusion criteria for clinical studies consisted of both retrospective and prospective study design and any ligament or tendon-to-bone repairs by ASAs with a minimum 1-year follow-up period. Evaluations were expected to be done by clinical-outcome measuring, radiologic findings and complication rates. The inclusion criteria for biomechanical studies consisted of all studies testing the biomechanical performance of ASAs on either cadaver and animal bones or synthetic surfaces. Test protocols might include soft tissue-to-bone repair models or the general performance of these anchors.

Exclusion criteria for this review were non-English manuscripts, review articles, commentaries, letters, case reports, and technical notes. For studies in which a kin relationship was identified, manuscripts were joined to make a single record so as to avoid repeating the same findings. One investigator selected studies for inclusion in the review. Articles were then independently assessed by a second investigator, who confirmed eligibility. The following variables were recorded in a predesigned database: general manuscript characteristics (author, year and journal), study design, testing protocols and findings for biomechanical studies,

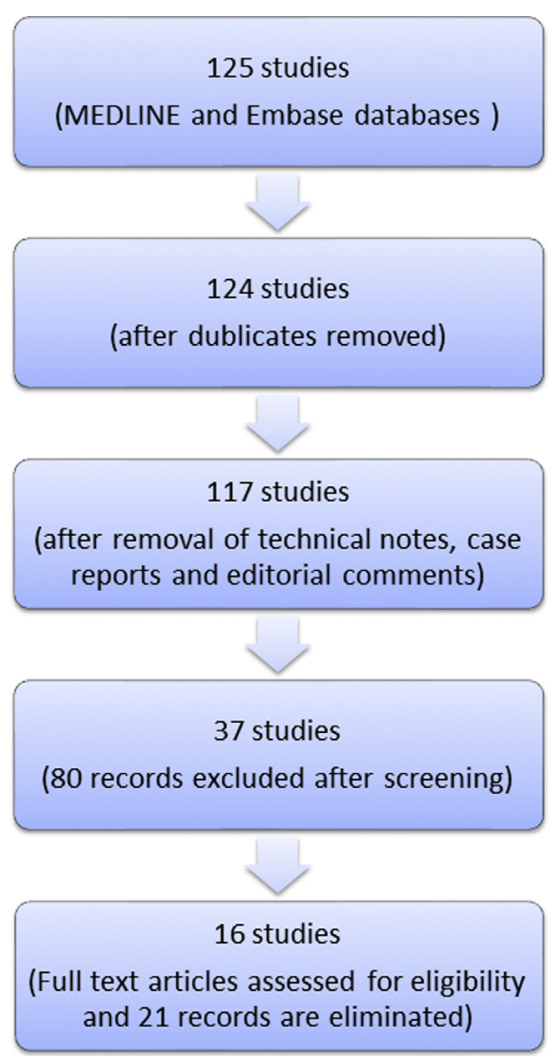

Fig 1. PRISMA flow diagram. From the initial 125 records, 37 full-text articles were reviewed for eligibility. Ultimately, 16 studies (13 experimental, 3 clinical and radiologic studies) were included.

imaging findings (tunnel widening, cyst formation), and clinical outcomes and complications (failure modes) for the clinical studies.

Biomechanical study data were reported quantitatively for cyclic loading, ultimate load to failure, displacement at failure, and stiffness-test results. Failure mode was qualitatively reported as anchor pullout, suture breaking, anchor breaking, and tendon tearing.

The quality of all included studies was independently evaluated using the Methodological Index for Nonrandomized Studies (MINORS) tool $^{20}$ (Appendix Table 1).

\section{Results}

The initial database search retrieved 125 titles and abstracts. After screening records for duplicates and inclusion criteria, 37 full-text articles were reviewed for eligibility. Ultimately, 16 studies (13 experimental studies, 3 clinical and radiologic studies) were included. The levels of evidence for the all 3 clinical studies were Level IV. The study's flow diagram is shown in Fig 1.

The mean quality rating of all biomechanical studies using the Methodological Index for Non-randomized Studies MINORS tool was $20.5 \pm 1.2$ points (of a maximum of 24 points, $85.2 \%$; range 19-22), mean 


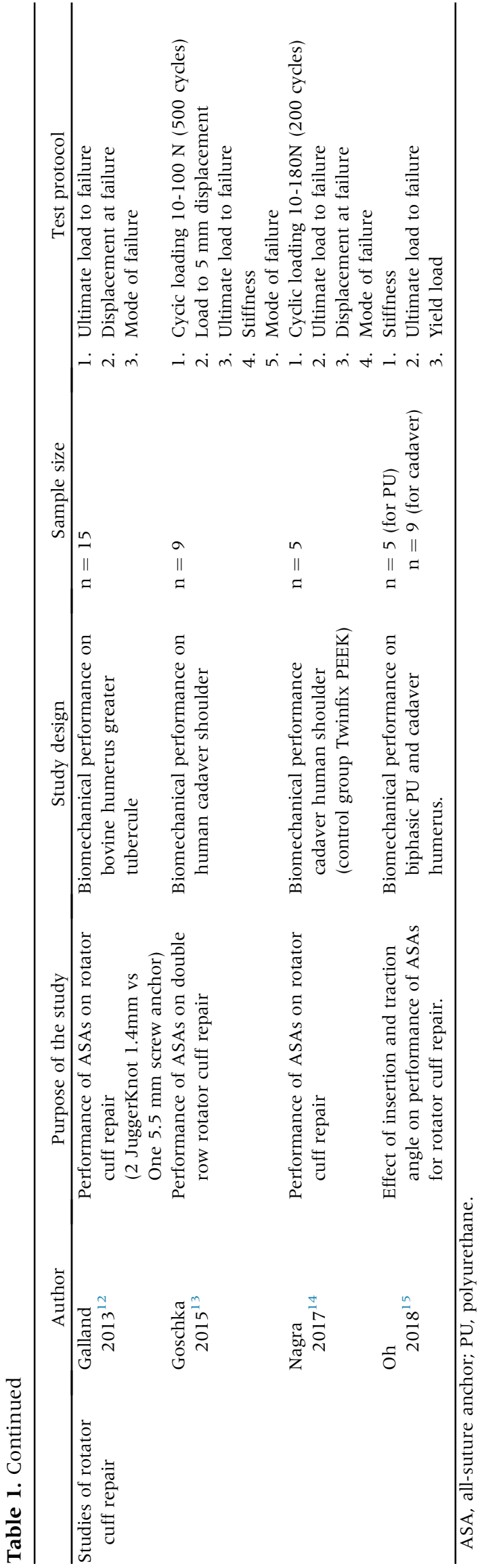

quality rating of all clinical studies was $13 \pm 1$ (of a maximum of 16 points, $81.2 \%$; range 12-14) (Appendix Table 2). The mean quality rating of all 16 studies was $84.5 \%$. The most common reason for point deductions was item 5, unbiased assessment of the study's endpoint. All except 2 studies (Pfeiffer et al., ${ }^{6}$ Willemot et al. $^{16}$ ) received 0 points for this item because the observers were not blinded to the study's endpoints.

Studies to measure biomechanical performance of ASAs are performed on various bone models: porcine cortical bone and distal femur metaphysis, ${ }^{3,4}$ bovine proximal tibia, ${ }^{5}$ bovine humerus greater tubercle, ${ }^{12}$ monophasic polyurethane (PU) foam, ${ }^{4,7}$ biphasic PU foam, ${ }^{15}$ cadaver glenoid, ${ }^{8}$ cadaver greater tubercle, ${ }^{13}$ and cadaver humerus to test biceps tenodesis. ${ }^{10,11}$ It is obvious that bone models and test protocols are not standardized, and both show high variability. Biomechanical test protocols measured the displacement under cyclic loading, displacement at failure, ultimate load to failure, stiffness, mode of failure, and load to $2 \mathrm{~mm}$ or $5 \mathrm{~mm}$ displacement (Table 1). Most of the biomechanical studies had a control suture anchor (screw anchor or polyetheretherketone [PEEK] anchor), and the results were compared.

Clinical studies were reviewed, and outcome data were reported according to clinical scores acquired from the Visual Analog Scale, the Western Ontario Shoulder Instability Index, the Disabilities of the Arm, Shoulder and Hand Score, and the Constant and Murley scoring systems. Imaging findings and complication rates were also reported in those clinical studies (Table 2).

ASA models used in the included studies were JuggerKnot 1.4, 1.5 and $2.9 \mathrm{~mm}$ (Biomet); Iconix 1, 2 and 3 (Stryker); Y-Knot 1.3, 1.8 and $2.9 \mathrm{~mm}$ (Conmed); Draw tight 1.8 and $3.2 \mathrm{~mm}$ (Parcus); Q-fix 1.8 and 2.8 mm, Suture Fix Ultra $1.7 \mathrm{~mm}$ (Smith \& Nephew); Omega Knot (ARC, Korea) (Table 2).

Cyclic loading tests showed that the least displacement was recorded with Q-Fix anchors. Also, failure mode was mostly by suture breaking for the Q-Fix, whereas anchor pullout was the most common for the others (Table 3). Four biomechanicals studies on cadaver glenoid bone compared the ASAs with a control bioabsorbable, biocomposite or PEEK anchor. In 2 of these studies, control anchors showed better results than ASAs (Y-Knot and JuggerKnot), ,6 but in the remaining 2 studies, ASAs (Suture-Fix Ultra and Q-Fix) showed better results than the control PEEK anchors despite their larger diameters than ASAs., Two studies simulating biceps tenodesis on cadavers both showed superior biomechanical results for control screw anchors when compared to ASAs (YKnot). ${ }^{10,11}$ Simulating the rotator cuff repair, 3 biomechanical studies compared ASAs with a control 
Table 2. Characteristics of the Included Clinical Studies

\begin{tabular}{|c|c|c|c|c|}
\hline $\begin{array}{c}\text { Study } \\
\end{array}$ & Purpose of the study & Study design and Level of Evidence & Sample size & Method \\
\hline Willemot $2016^{16}$ & $\begin{array}{l}\text { Radiologic and clinical outcomes } \\
\text { arthroscopic labral repairs with ASAs. }\end{array}$ & $\begin{array}{l}\text { Postoperative MRI scan } \\
\text { DASH, Constant and WOSI } \\
\text { Level of Evidence: IV }\end{array}$ & $\begin{array}{c}\mathrm{n}=20 \text { patients } \\
58 \text { anchors }\end{array}$ & $\begin{array}{l}\text { Radiologic appearance of bone at the } \\
\text { anchor site was judged by the presence } \\
\text { of cyst formation, tunnel widening (> } \\
2 \mathrm{~mm} \text { ) or bone edema. } \\
\text { Clinical scores }\end{array}$ \\
\hline Byrd $2017^{17}$ & $\begin{array}{l}\text { Incidence of intraoperative pull-out of } \\
\text { ASAs for acetabular labral repairs. }\end{array}$ & $\begin{array}{l}\text { Retrospective review of intraoperative } \\
\text { anchor failure incidence } \\
\text { Level of Evidence: IV }\end{array}$ & $\begin{array}{l}434 \text { patient } \\
2007 \text { anchors }\end{array}$ & $\begin{array}{l}\text { (1) The age and gender of all cases; (2) } \\
\text { the number of cases in which labral } \\
\text { repair was performed; (3) the number } \\
\text { of anchors used; (4) the number of } \\
\text { cases in which intraoperative anchor } \\
\text { failure occurred; (5) the number of } \\
\text { anchors that failed; and (6) the age } \\
\text { and gender of those cases in which } \\
\text { anchor failure occurred }\end{array}$ \\
\hline Van Der Bracht $2018^{18}$ & $\begin{array}{l}\text { Clinical and radiologic } \\
\text { (at leaset } 1 \text { year later) study to } \\
\text { investigate the feasibility and safety of } \\
\text { ASAs in arthroscopic rotator cuff } \\
\text { repair }\end{array}$ & $\begin{array}{l}\text { Prospective cohort; } \\
\text { VAS score (for pain and satisfaction), } \\
\text { constant Murley score, strength of SS } \\
\text { muscle by dynamometer and } \\
\text { radiologic findings } \\
\text { Level of evidence: IV }\end{array}$ & $\begin{array}{l}\mathrm{n}=20 \text { patients } \\
48 \text { anchors }\end{array}$ & $\begin{array}{l}\text { Integrity of the cuff repair, cyst formation } \\
\text { around anchor, ingrowth of the bone } \\
\text { into the anchor, and integrity of the } \\
\text { bone tunnel border were evaluated. } \\
\text { Clinical scores }\end{array}$ \\
\hline
\end{tabular}




\begin{tabular}{|c|c|c|c|c|c|c|c|c|}
\hline & Author & Anchor & Cyclic loading & $\begin{array}{l}\text { Ultimate load to } \\
\text { failure }\end{array}$ & $\begin{array}{l}\text { Displace-ment at } \\
\text { failure }\end{array}$ & $\begin{array}{l}\text { Stiff-ness } \\
\mathrm{N} / \mathrm{mm}\end{array}$ & Mode of failure & \\
\hline \multirow[t]{10}{*}{$\begin{array}{r}\text { Studies of } \\
\text { Glenoid }\end{array}$} & \multirow[t]{5}{*}{$\begin{array}{c}\text { Barber FA } \\
2013 \text { and } \\
2017^{3,4}\end{array}$} & $\begin{array}{l}\text { JuggerKnot } 1.4- \\
1.5-2.9\end{array}$ & $\begin{array}{l}\text { Porcine bone: } \\
\text { 1.5: } 1.39 \mathrm{~mm} \\
\text { 2.9: } 1.44 \mathrm{~mm}\end{array}$ & $\begin{array}{l}\text { Porcine bone: } \\
239 \mathrm{~N}-290 \mathrm{~N}- \\
519 \mathrm{~N}\end{array}$ & $\begin{array}{l}\text { Porcine bone: } \\
0.22 \mathrm{~mm}-0.22 \\
\mathrm{~mm}-0.22 \mathrm{~mm}\end{array}$ & $198-57-76$ & $\begin{array}{l}\text { 1.4: Anchor pullout and } \\
\text { Suture break } \\
1.5-2.9: \text { Suture break } \\
\text { only }\end{array}$ & \multirow{5}{*}{$\begin{array}{c}\text { Result } \\
\text { Ultimate load at failure was } \\
\text { correlated directly with the number } \\
\text { of sutures. } \\
\text { Y-Knot demonstrated greater } \\
\text { displacement than the JuggerKnot } \\
\text { and Q-Fix } \\
\text { Both JuggerKnot }(81 \%) \text { and Q-Fix } \\
(97 \%) \text { anchors failed predominantly } \\
\text { by the suture breakage; however, Y } \\
\text { knot had high anchor pull out rate. } \\
\text { None of the all-suture anchors } \\
\text { reached a clinically significant } 5 \text { mm } \\
\text { displacement during cyclic loading. }\end{array}$} \\
\hline & & $\begin{array}{l}\text { Iconix } \\
1-2-3\end{array}$ & $\begin{array}{l}\text { Porcine bone: } \\
1.87 \mathrm{~mm}- \\
1.55 \mathrm{~mm}- \\
1.44 \mathrm{~mm}\end{array}$ & $\begin{array}{l}\text { Porcine bone: } \\
209 \mathrm{~N}-469 \mathrm{~N}- \\
570 \mathrm{~N} \\
\text { Biphasic PU foam: } \\
235 \mathrm{~N}-520 \mathrm{~N}\end{array}$ & $\begin{array}{l}\text { Porcine bone: } \\
0.31 \mathrm{~mm}-0.23 \\
\mathrm{~mm}-0.20 \mathrm{~mm} \\
\text { Biphasic PU foam: } \\
0.23 \mathrm{~mm}-0.43 \\
\mathrm{~mm}\end{array}$ & $65-83-89$ & $\begin{array}{l}\text { 1: Anchor andsuture } \\
\text { break } \\
\text { 2: Mostly suture break } \\
\text { 3: Mostly anchor break }\end{array}$ & \\
\hline & & $\begin{array}{l}\text { Y knot } \\
\quad 1.3-1.8-2.9\end{array}$ & $\begin{array}{l}\text { Porcine bone: } \\
2.4 \mathrm{~mm} \mathrm{-} \\
2.0 \mathrm{~mm} \mathrm{-} \\
3.52 \mathrm{~mm}\end{array}$ & $\begin{array}{l}\text { Porcine bone: } \\
250 \mathrm{~N}-477 \mathrm{~N}- \\
603 \mathrm{~N} \\
\text { Biphasic PU foam: } \\
\text { 152N - 531N - } \\
657 \mathrm{~N}\end{array}$ & $\begin{array}{l}\text { Porcine bone: } \\
0.45 \mathrm{~mm}-0.33 \\
\mathrm{~mm}-0.55 \mathrm{~mm} \\
\text { Biphasic PU foam: } \\
*- \\
0.23 \mathrm{~mm}- \\
0.19 \mathrm{~mm}\end{array}$ & $65-74-84$ & $\begin{array}{l}\text { All: High anchor pullout } \\
\text { rate }\end{array}$ & \\
\hline & & $\begin{array}{l}\text { Draw Tight } 1.8- \\
\quad 3.2\end{array}$ & $\begin{array}{l}\text { Porcine bone: } \\
\quad 2.12 \mathrm{~mm}-2.62 \\
\mathrm{~mm}\end{array}$ & $\begin{array}{l}\text { Porcine bone: } \\
\text { 290N - 418N } \\
\text { Biphasic PU foam: } \\
263 \mathrm{~N}-191 \mathrm{~N}\end{array}$ & $\begin{array}{l}\text { Porcine bone: } \\
0.30 \mathrm{~mm}-0.30 \\
\text { mm } \\
\text { Biphasic PU foam: } \\
0.24 \mathrm{~mm}- \\
0.20 \mathrm{~mm}\end{array}$ & $41-49$ & $\begin{array}{l}\text { All: High anchor pullout } \\
\text { rate }\end{array}$ & \\
\hline & & $\begin{array}{l}\text { Q Fix } \\
\quad 1.8-2.8\end{array}$ & $\begin{array}{l}\text { Porcine bone: } \\
1.22 \mathrm{~mm}- \\
1.58 \mathrm{~mm}\end{array}$ & $\begin{array}{l}\text { Porcine bone: } \\
\text { 346N - 495N } \\
\text { Biphasic PU foam: } \\
\text { 292N - 495N }\end{array}$ & $\begin{array}{l}\text { Porcine bone: } \\
0.19 \mathrm{~mm}-0.23 \\
\text { mm } \\
\text { Biphasic PU foam: } \\
0.11 \mathrm{~mm}- \\
0.16 \mathrm{~mm}\end{array}$ & $55-57$ & $\begin{array}{l}\text { All: Suture break (1 } \\
\text { anchor pullout) }\end{array}$ & \\
\hline & \multirow[t]{3}{*}{$\begin{array}{l}\text { Dwyer } \\
2014^{5}\end{array}$} & $\begin{array}{l}\text { YKnot } 1.3 \mathrm{~mm} \\
\text { (Handset) } \\
\text { (bovine tibia/ } \\
\text { human glenoid) }\end{array}$ & - & $140 \mathrm{~N} / 91 \mathrm{~N}$ & $\begin{array}{l}\text { Displacement at } 50 \mathrm{~N}: \\
\quad 4.6 \mathrm{~mm} / 7.5 \mathrm{~mm}\end{array}$ & $8.7 / 4.3$ & $\begin{array}{l}\text { The primary mode of } \\
\text { failure in all-suture } \\
\text { anchors was } \\
\text { anchor pullout }\end{array}$ & \multirow{3}{*}{$\begin{array}{l}\text { Pretensioning the YKnot } \\
\text { to } 60 \mathrm{~N} \text { ensures that the anchor is } \\
\text { well fixed, consistently eliminating } \\
\text { laxity and displacement in both high- } \\
\text { density bovine and lower-density } \\
\text { cadaveric bone } \\
\text { Some anchor pullout was seen below } \\
\text { the } 60 \mathrm{~N} \text { set as the } \\
\text { pretensioning force }\end{array}$} \\
\hline & & $\begin{array}{l}\text { YKnot }(60 \mathrm{~N} \\
\text { Pretensioned) } \\
\text { (bovine tibia/ } \\
\text { human glenoid) }\end{array}$ & - & $135 \mathrm{~N} / 145 \mathrm{~N}$ & $\begin{array}{l}\text { Displacement at } 50 \mathrm{~N}: \\
1.9 \mathrm{~mm} / 1.9 \mathrm{~mm}\end{array}$ & $21 / 21.7$ & $\begin{array}{l}\text { The primary mode of } \\
\text { failure in all-suture } \\
\text { anchors was anchor } \\
\text { pullout }\end{array}$ & \\
\hline & & $\begin{array}{l}\text { Bio Mini Revo } 3.1 \\
\text { mm (control) }\end{array}$ & & $206 \mathrm{~N} / 107 \mathrm{~N}$ & $\begin{array}{l}\text { Displacement at } 50 \mathrm{~N} \text { : } \\
3.5 \mathrm{~mm} / 2.7 \mathrm{~mm}\end{array}$ & $12.8 / 14.4$ & $\begin{array}{l}6 \text { anchor pullout, } 2 \text { eyelet } \\
\text { failure }\end{array}$ & \\
\hline & \multirow[t]{2}{*}{$\begin{array}{l}\text { Pfeiffer } \\
2014^{6}\end{array}$} & $\begin{array}{l}\text { JuggerKnot } 1.4 \\
\mathrm{~mm}\end{array}$ & $2.9 \mathrm{~mm}$ & $141 \mathrm{~N}$ & $13.7 \mathrm{~mm}$ & - & Anchor pullout (8) & $\begin{array}{l}\text { Consistent cavity formation, } \\
\text { significant }\end{array}$ \\
\hline & & $\begin{array}{l}\text { Control 2.4-mm } \\
\text { BioComposite } \\
\text { SutureTak }\end{array}$ & $1.3 \mathrm{~mm}$ & $136.7 \mathrm{~N}$ & $3.2 \mathrm{~mm}$ & - & $\begin{array}{l}\text { Anchor pullout (5) and } \\
\text { breakage of the top } \\
\text { portion of } \\
\text { the anchor (3) }\end{array}$ & $\begin{array}{l}\text { expansion of the drill tunnel, was } \\
\text { associated with the JuggerKnot } \\
\text { anchors in the canine glenoid. All } \\
\text { sutures incite foreign body reactions } \\
\text { to varying degrees wherever they are } \\
\text { placed in the body } \\
\text { JuggerKnot anchor was slipping }\end{array}$ \\
\hline
\end{tabular}




\begin{tabular}{|c|c|c|c|c|c|c|c|}
\hline Author & Anchor & Cyclic loading & $\begin{array}{l}\text { Ultimate load to } \\
\text { failure }\end{array}$ & $\begin{array}{l}\text { Displace-ment at } \\
\text { failure }\end{array}$ & $\begin{array}{l}\text { Stiff-ness } \\
\mathrm{N} / \mathrm{mm}\end{array}$ & Mode of failure & Result \\
\hline \multirow{7}{*}{$\begin{array}{l}\text { Douglass } \\
2017^{7}\end{array}$} & & & & & & & $\begin{array}{c}\text { within the prepared hole before } \\
\text { failure. Clinically, such slipping leads } \\
\text { to a loss of reduction and can hinder } \\
\text { healing. } \\
\text { Based on the biomechanical findings } \\
\text { in human bone and histologic } \\
\text { findings in canine subjects, ASAs } \\
\text { may be at risk for clinical failure }\end{array}$ \\
\hline & $\begin{array}{l}\text { JuggerKnot } 1.4- \\
\quad 1.5-2.9\end{array}$ & - & $\begin{array}{l}20 \text { pcf: JuggerKnot } \\
\text { 2.9: } 194 \mathrm{~N} \\
\text { 30pcf: } \\
\text { JuggerKnot2.9: } \\
\text { 301N }\end{array}$ & - & - & $\begin{array}{l}20 \text { pcf:Anchor pullout } \\
30 \text { pcf: Anchor pullout }\end{array}$ & $\begin{array}{l}\text { ASAs exhibited less displacement } \\
\text { and greater maximum loads in } \\
\text { higher density (30pcf) bone } \\
\text { substitute. } \\
\text { The cyclic displacement and }\end{array}$ \\
\hline & $\begin{array}{l}\text { Iconix } 1-2-25- \\
\quad 3\end{array}$ & - & $\begin{array}{l}20 \text { pcf: } \\
\text { Iconix 2: } 163 \mathrm{~N} \\
\text { Iconix 25: } 196 \mathrm{~N} \\
\text { (highest) } \\
\text { Iconix 3: } 180 \mathrm{~N} \\
30 \text { pcf: } \\
\text { Iconix 25: } 307 \mathrm{~N} \\
\text { Iconix 3: } 276 \mathrm{~N}\end{array}$ & - & - & $\begin{array}{l}20 \text { pcf:Anchor pullout } \\
30 \text { pcf: Anchor pullout }\end{array}$ & $\begin{array}{c}\text { maximum load of ASAs vary widely } \\
\text { depending on anchor design and } \\
\text { bone density } \\
\text { Q-Fix 1.8, however, performed } \\
\text { better than all other anchors in } \\
\text { displacement and had maximum } \\
\text { failure loads comparable with the } \\
\text { highest values of the other anchors }\end{array}$ \\
\hline & $\begin{array}{l}\text { Y-Knot } \\
\quad 1.3-1.8\end{array}$ & - & $\begin{array}{l}20 \text { pcf: } \\
\quad \text { YKnot } 1.8: 176 \mathrm{~N}\end{array}$ & - & - & $\begin{array}{l}20 \text { pcf:Anchor pullout } \\
30 \text { pcf: Anchor pull out }\end{array}$ & $\begin{array}{l}\text { tested. } \\
\text { Faiulre mode is mostly anchor }\end{array}$ \\
\hline & $\begin{array}{l}\text { Suture Fix Ultra } \\
\quad 1.7\end{array}$ & - & & - & - & $\begin{array}{l}20 \text { pcf:Anchor pullout } \\
30 \text { pcf: Anchor pull out }\end{array}$ & $\begin{array}{l}\text { pullout, probably due to monphasic } \\
\text { structure of the PU substitute. But }\end{array}$ \\
\hline & $\begin{array}{r}\text { Q Fix } \\
1.8\end{array}$ & $\begin{array}{l}\text { Least } \\
\text { displacement in } \\
\text { both } 20 \text { and } 30 \\
\text { pcf }(0.1 \mathrm{~mm} \\
\text { after } 200 \text { cycles, } \\
0.2 \mathrm{~mm} \text { after } \\
400 \text { cycles })\end{array}$ & 30 pcf: $291 \mathrm{~N}$ & $\begin{array}{l}\text { Best result (least } \\
\text { displacement) in } \\
\text { both } 20 \text { pcf and } \\
30 \text { pcf }\end{array}$ & - & $\begin{array}{l}20 \text { pcf:Anchor pullout } \\
30 \text { pcf: Suture break }\end{array}$ & $\begin{array}{c}\text { this situtation changed with Q fix } \\
\text { ancor on 30pcf substitute (suture } \\
\text { break) }\end{array}$ \\
\hline & $\begin{array}{l}\text { Bioraptor PEEK } \\
2.3 \mathrm{~mm} \\
\text { (Control) }\end{array}$ & $\begin{array}{l}0.3 \mathrm{~mm}(20 \mathrm{pcf}) \\
0.4 \mathrm{~mm}(30 \mathrm{pcf}) \\
(200 \text { cycles })\end{array}$ & - & - & - & - & \\
\hline \multirow[t]{3}{*}{$\begin{array}{l}\text { Erickson } \\
2017^{8}\end{array}$} & JuggerKnot 1.4 & - & $171.5 \mathrm{~N}$ & $\begin{array}{l}\text { Load to } 2 \mathrm{~mm} \\
\quad \text { displacement: } 36 \mathrm{~N}\end{array}$ & - & - & $\begin{array}{l}\text { A second-generation all-soft suture } \\
\text { anchor (Suture Fix Ultra) showed }\end{array}$ \\
\hline & $\begin{array}{l}\text { Suture Fix Ultra } \\
\quad 1.7\end{array}$ & - & $182.5 \mathrm{~N}$ & $\begin{array}{l}\text { Load to } 2 \mathrm{~mm} \\
\quad \text { displacement: } 42 \mathrm{~N}\end{array}$ & - & - & $\begin{array}{l}\text { greater loads to } 2 \mathrm{~mm} \text { of } \\
\text { displacement than a first-generation }\end{array}$ \\
\hline & $\begin{array}{l}\text { Bioraptor PEEK } \\
2.3 \mathrm{~mm} \\
\text { (control) }\end{array}$ & - & $132 \mathrm{~N}$ & $\begin{array}{l}\text { Load to } 2 \mathrm{~mm} \\
\quad \text { displacement: } 39 \mathrm{~N}\end{array}$ & - & - & $\begin{array}{l}\text { all-soft suture anchor (JuggerKnot). } \\
\text { Both all-soft suture anchors had } \\
\text { higher load to failure than PEEK } \\
\text { anchor (Bioraptor 2.3 PK). }\end{array}$ \\
\hline $\begin{array}{l}\text { Ruder } \\
\quad 2018^{9}\end{array}$ & JuggerKnot 1.5 & $\begin{array}{l}21 \mathrm{~mm}: 2.50 \\
17 \mathrm{~mm}: 1.70 \\
13 \mathrm{~mm}: 1.13\end{array}$ & $\begin{array}{l}21 \mathrm{~mm}: 194 \mathrm{~N} \\
17 \mathrm{~mm}: 190 \mathrm{~N} \\
13 \mathrm{~mm}: 138 \mathrm{~N}\end{array}$ & - & - & $\begin{array}{l}\text { High clinical failure }(3 \mathrm{~mm} \\
\text { and } 5 \mathrm{~mm} \text { ) with } 21 \mathrm{~mm} \\
\text { depth. No clinical } \\
\text { failure with } 13 \mathrm{~mm} \text {. }\end{array}$ & $\begin{array}{l}\text { İnserting the anchor at a depth of } \\
17 \mathrm{~mm} \text { reduced the displacement } \\
\text { after cyclic loading without } \\
\text { reducing the ultimate load to } \\
\text { failure. }\end{array}$ \\
\hline
\end{tabular}




\begin{tabular}{|c|c|c|c|c|c|c|c|c|}
\hline & Author & Anchor & Cyclic loading & $\begin{array}{l}\text { Ultimate load to } \\
\text { failure }\end{array}$ & $\begin{array}{l}\text { Displace-ment at } \\
\text { failure }\end{array}$ & $\begin{array}{l}\text { Stiff-ness } \\
\mathrm{N} / \mathrm{mm}\end{array}$ & Mode of failure & Result \\
\hline \multirow[t]{2}{*}{$\begin{array}{l}\text { Studies } \\
\text { on Biceps } \\
\text { tenodesis }\end{array}$} & $\begin{array}{l}\text { Chiang } \\
2016^{10}\end{array}$ & $\begin{array}{l}\text { Y-Knot } 1.3 \mathrm{~mm} / \\
\text { Milagro } \\
\text { Bioreplaceable } \\
\text { Screw } 8 \times 23 \\
\text { mm }\end{array}$ & $\begin{array}{l}8.1 \mathrm{~mm} / \text { (Control } \\
3.4 \mathrm{~mm} \text { ) }\end{array}$ & $\begin{array}{l}\text { 239N/(control } 254.4 \\
\mathrm{~N})\end{array}$ & $\begin{array}{l}20.3 \mathrm{~mm} / \\
\quad(\text { control } 13.3 \mathrm{~mm})\end{array}$ & $\begin{array}{l}26 /(\text { control } \\
27.7)\end{array}$ & $\begin{array}{l}\text { ASA: Anchor pullout (n: } \\
\text { 4), Tendon tear (n: } 4) \\
\text { Control: (screw pullout } \\
\text { (2), tendon tear (6) }\end{array}$ & $\begin{array}{l}\text { ASA technique displayed values of } \\
\text { ultimate failure load and stiffness } \\
\text { comparable to that of the } \\
\text { interference screw technique. } \\
\text { However, the cyclic and failure } \\
\text { displacement values of the } \\
\text { interference screw trials were } \\
\text { significantly less than that of the } \\
\text { ASA }\end{array}$ \\
\hline & $\begin{array}{l}\text { Hong } \\
2018^{11}\end{array}$ & $\begin{array}{l}\text { Y-Knot } 1.3 \mathrm{~mm} / \\
\quad \text { Milagro } \\
\text { Bioreplaceable } \\
\text { Screw } 8 \times \\
23 \mathrm{~mm}\end{array}$ & $\begin{array}{l}6.4 \mathrm{~mm} /(\text { control: } \\
\quad 3.7 \mathrm{~mm})\end{array}$ & $\begin{array}{l}186.6 \mathrm{~N} / \text { (control } \\
203.8 \mathrm{~N} \text { ) }\end{array}$ & $\begin{array}{l}16.3 \mathrm{~mm} / \text { (control } \\
13.3 \mathrm{~mm} \text { ) }\end{array}$ & $\begin{array}{l}26.1 / \\
\text { (control: } \\
27.1)\end{array}$ & $\begin{array}{l}\text { ASA: Anchor pullout } \\
\text { (n:6), Tendon tear } \\
(\mathrm{n}: 2) \\
\text { Control: (screw pullout } \\
\text { (0), Tendon tear (8) }\end{array}$ & $\begin{array}{l}\text { ASA technique displayed values of } \\
\text { ultimate failure load, failure } \\
\text { displacement and stiffness } \\
\text { comparable to that of the } \\
\text { interference screw technique. } \\
\text { However, the cyclic displacement } \\
\text { values of the interference screw } \\
\text { trials were significantly less than } \\
\text { that of the ASA }\end{array}$ \\
\hline \multirow[t]{7}{*}{$\begin{array}{l}\text { Studies of } \\
\text { rotator } \\
\text { cuff } \\
\text { repair }\end{array}$} & $\begin{array}{l}\text { Galland } \\
2013^{12}\end{array}$ & $\begin{array}{l}\text { JuggerKnot } 1.4 \\
\text { mm X2 / } \\
\text { (Control Screw } \\
\text { Anchor } 5.5 \\
\text { mm) }\end{array}$ & - & $\begin{array}{l}\text { ASA }(\mathrm{X} 2): 265 \mathrm{~N} \\
\quad / \text { control: } 325 \mathrm{~N} \\
(P: 0.09)\end{array}$ & $\begin{array}{l}\text { ASA (X2): } 23 \mathrm{~mm} \\
\text { /control: } 21 \mathrm{~mm} \\
(P: 0.46)\end{array}$ & - & $\begin{array}{l}\text { ASA: } 12 \text { anchor pullout, } 1 \\
\text { thread fracture/control } \\
\text { screw anchor: } 8 \text { anchor } \\
\text { pullout, } 5 \text { eyelet } \\
\text { fractures }\end{array}$ & $\begin{array}{l}\text { There was no statistically significant } \\
\text { difference between pullout } \\
\text { strength and displacement of a } \\
\text { double-fixed bone ASA and a } \\
\text { single-fixed control SA }\end{array}$ \\
\hline & $\begin{array}{l}\text { Goschka } \\
2015^{13}\end{array}$ & $\begin{array}{l}\text { Medial row/ } \\
\text { lateral row }\end{array}$ & & & & & & $\begin{array}{l}\text { The biomechanical performance of } \\
\text { anchor configurations using the }\end{array}$ \\
\hline & & Iconix $2 /$ Iconix 2 & - & $313.2 \mathrm{~N}$ & - & - & $\begin{array}{l}\text { Anchor pullout: } 5 \\
\text { Suture tear: } 3 \\
\text { Knot slippage: } 1\end{array}$ & $\begin{array}{l}\text { ICONIX2 would be comparable to } \\
\text { that of the configuration of solid- } \\
\text { body anchors, as no significant }\end{array}$ \\
\hline & & $\begin{array}{l}\text { Iconix2 / ReelX } \\
3.9\end{array}$ & - & $457.9 \mathrm{~N}$ & - & - & $\begin{array}{l}\text { Anchor pullout: } 6 \\
\text { Suture tear: } 2 \\
\text { Suture pull out of } \\
\text { anchor: } 1\end{array}$ & $\begin{array}{l}\text { differences were found between } \\
\text { groups for any metric tested. } \\
\text { (No significant change in between } \\
\text { groups for all tests) }\end{array}$ \\
\hline & & Iconix2/ReelX 4.5 & $\begin{array}{l}\text { Max gap } \\
\text { formation in } \\
\text { anterior } \\
\text { anchors } \\
\text { Min gap } \\
\text { formation in } \\
\text { posterior } \\
\text { anchors }\end{array}$ & $420.2 \mathrm{~N}$ & - & - & $\begin{array}{l}\text { Anchor pullout: } 6 \\
\text { Suture tear: } 1 \\
\text { Suture pullout of } \\
\text { anchor: } 1 \\
\text { Eyelet break: } 1\end{array}$ & \\
\hline & & $\begin{array}{l}\text { Control: } \\
\text { CorkScrew4.5/ } \\
\text { SwiveLock } 4.75\end{array}$ & $\begin{array}{l}\text { Max gap } \\
\text { formation in } \\
\text { posterior } \\
\text { anchors } \\
\text { Min. gap } \\
\text { formation in } \\
\text { anterior } \\
\text { anchors }\end{array}$ & $430.9 \mathrm{~N}$ & - & - & $\begin{array}{l}\text { Anchor pullout: } 4 \\
\text { Suture tear: } 1 \\
\text { Suture pullout of } \\
\text { anchor: } 2 \\
\text { Muscle tear: } 2\end{array}$ & \\
\hline & & & 100 cycles & $137.8 \mathrm{~N}$ & $33.7 \mathrm{~mm}$ & & & \\
\hline
\end{tabular}




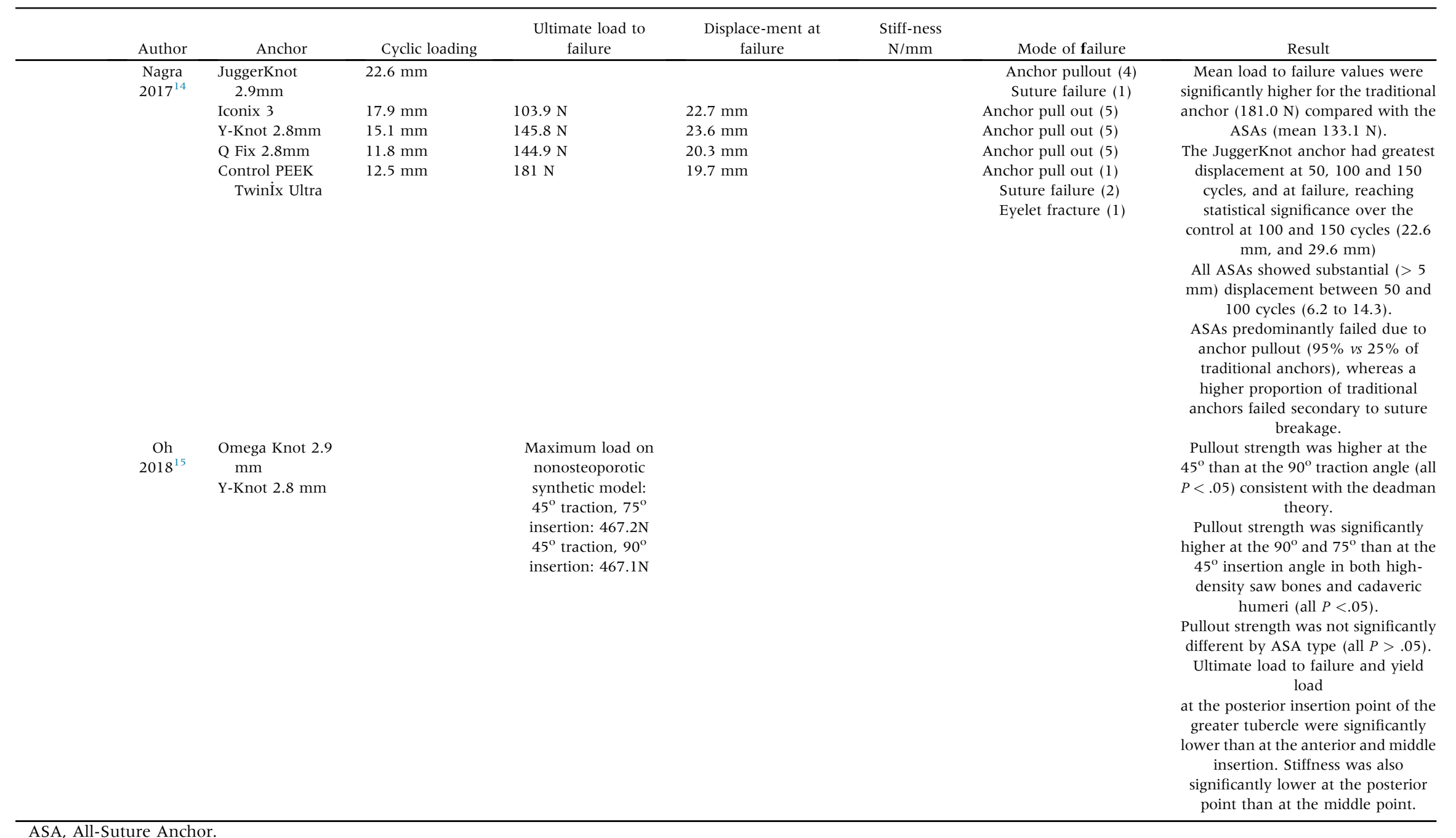




\begin{tabular}{|c|c|c|c|c|c|}
\hline Study & Anchor/Fixation & Clinical outcome & Radiologic findings & Complications & Result \\
\hline Willemot $2016^{16}$ & $\begin{array}{l}\text { JuggerKnot } 1.4 \mathrm{~mm} \\
\text { Labral repiar }\end{array}$ & $\begin{array}{l}\text { Satisfactory clinical results: } \\
\text { WOSI: } 70.6, \\
\text { DASH: } 18.9 \\
\text { Constant: } 89.3 \\
\text { No recurrence of } \\
\text { instability. }\end{array}$ & $\begin{array}{l}\text { None of the patients displayed } \\
\text { large cyst formation. } \\
\text { Small cysts (grade } 3 \text { ) were } \\
\text { found in } 2 \text { patients ( } 2 \text { anchors). } \\
\text { Tunnel widening (grade } 2 \text { ) was } \\
\text { apparent in } 3 \text { patients ( } 3 \\
\text { anchors) with an average } \\
\text { widening of } 3.3 \text { mm (range } 3-4 \\
\text { mm). } \\
\text { Bone edema (grade 1) at the } \\
\text { anchor-site was seen in } 6 \\
\text { patients ( } 8 \text { anchors). } \\
\text { The remaining } 9 \text { patients ( } 45 \\
\text { anchors) did not display } \\
\text { reactive bone changes. }\end{array}$ & No complications & $\begin{array}{l}\text { Promising early radiographic and } \\
\text { clinical outcome after } \\
\text { arthroscopic glenohumeral } \\
\text { labral repair using all-suture } \\
\text { anchors. In this cohort of } 20 \\
\text { patients with } 58 \text { anchors and a } \\
\text { mean follow-up of } 19 \text { months, } \\
\text { bone reactions were few and } \\
\text { low grade on the postoperative } \\
\text { MRIs, independent of anchor } \\
\text { position. Clinical scores } \\
\text { demonstrate satisfactory } \\
\text { functional outcomes without } \\
\text { recurrence of subluxation or } \\
\text { dislocation. }\end{array}$ \\
\hline Byrd $2017^{17}$ & $\begin{array}{l}\text { Q Fix } 1.8 \mathrm{~mm} \\
\text { Acetabular labral repair }\end{array}$ & - & & $\begin{array}{l}\text { A total of } 33 \text { anchors pulled } \\
\text { out among } 30 \text { patients, } \\
\text { representing a } 1.6 \% \\
\text { incidence among all } \\
\text { anchors. No statistically } \\
\text { significant difference } \\
\text { compared with the patient } \\
\text { population in which no } \\
\text { anchor pulled out. } \\
\text { Most common reason for } \\
\text { failure was to have the } \\
\text { anchor securely imbedded } \\
\text { in bone. }\end{array}$ & $\begin{array}{l}\text { An overall failure rate of } 1.6 \% \\
\text { seems quite acceptable. }\end{array}$ \\
\hline $\begin{array}{l}\text { Van Der Bracht } \\
2018^{18}\end{array}$ & $\begin{array}{l}\text { JuggerKnot } 2.9 \mathrm{~mm} \\
\text { Double row rotator cuff } \\
\text { repair } \\
\text { (Both ASA) }\end{array}$ & $\begin{array}{l}\text { VAS for pain: } 6.88 \text { to } 2.12 \\
\text { VAS for satisfaction: } 9.18 \\
\text { Post op constant: } 79.05 \\
\text { No difference in SS } \\
\text { muscle strength } \\
\text { between operated and } \\
\text { nonoperated sides }\end{array}$ & $\begin{array}{l}1 \text { retear, } 5 \text { small tears at } \\
\text { musculotendinous junction } \\
\text { Local fluid collection (not } \\
\text { encapsulated) } 10.4 \%(89.6 \% \\
\text { no fluid around anchor) } \\
\text { No cyst formation (fluid } \\
\text { diameter twice the anchor } \\
\text { diameter) } \\
\text { Thin, uninterrupted tunnel wall }\end{array}$ & $\begin{array}{l}1 \text { deep wound infection } \\
\text { One repeat arthroscopy } 61 \\
\text { days later (1 loose lateral } \\
\text { anchor) }\end{array}$ & $\begin{array}{l}\text { No fluid could be detected } \\
\text { between the anchors and the } \\
\text { edge of the bony tunnel for } \\
90 \% \text { of the anchors. } \\
\text { Full rotator cuff integrity was } \\
\text { seen in } 19 \text { patients, with only } 1 \\
\text { patient sustaining a retear. } \\
\text { Clinical results are comparable } \\
\text { with an arthroscopic rotator } \\
\text { cuff repair using classic anchors. } \\
\text { Study shows promising early } \\
\text { radiographic and clinical results } \\
\text { after arthroscopic rotator cuff } \\
\text { repair using ASAs. }\end{array}$ \\
\hline
\end{tabular}


screw or PEEK anchor. Although 2 of these studies $^{12,13}$ did not find any significant difference between groups, 1 study ${ }^{14}$ found better biomechanical results for control PEEK anchor when compared to ASAs (Q-Fix, JuggerKnot, Iconix, and Y-Knot). Test results, especially the failure mode, relied on the bone model. The cadaver humerus greater tubercle seemed to be less durable with the ASAs than other bone models, and failure mode is more likely to be by anchor pull out (Table 3).

Studies investigating clinical and radiologic findings were very few, and only 3 case series ( 2 prospective, 1 restrospective) were included in this review (Table 2 and Table 4). ${ }^{16-18}$ Each study had a different surgical repair modality for differing anatomic locations (gleno-humeral labral repair, ${ }^{16}$ acetabular labral repair ${ }^{17}$ and rotator cuff tendon repair ${ }^{18}$ ). Clinical failure was found to occur seldomly, anchor pullout was seen only in a rotator cuff repair study group in l patient in 1 anchor $(n=48)$. According to radiologic findings, cyst formation around the anchor was seen only in the gleno-humeral labral repair study group and included only 2 anchors $(n=58)$.

\section{Discussion}

This systematic review found that ASAs have similar biomechanical properties when compared with metal screw, bioabsorbable, biocomposite, or PEEK anchors. However, only 3 level IV clinical studies were identified; consequently, insufficient data are currently available to draw conclusions about clinical outcomes.

With this sytematic review, it has been demonstrated that the biomechanical performance of ASAs is affected primarily by the bone model. Synthetic PU foam is a commonly used model for ASA testing. Douglass et al. used 2 different monophasic PU foam models to test ASAs and found better biomechanical results with increasing density. ${ }^{7}$ The Q-Fix, especially, showed less displacement with cyclic loading and changed the mode of failure from anchor pullout to suture breaking by increasing the PU density from 20 pound-force per cubic foot (pcf) to $30 \mathrm{pcf}$. Barber et al. advocated the usage of this biphasic artificial PU model to mimic cancellous (12 pcf PU foam) and cortical bone (fiber-filled epoxy coating). ${ }^{4}$ They tested and compared different types of ASAs in the biphasic PU as well as in porcine femur cortical bone. They found similar test results for each type of ASA in both models. These results supported the use of biphasic PU models for experimental studies.

On the other hand, differing mechanical results were also reported in cadaver studies. Even in the same cadaver region, different insertion sites for the ASAs would end up with very different results. These differences can be explained by the bone density at the insertion site. Cortical bone density seems to be the main determinant of the initial fixation strength of an ASA. Placing an ASA in an area with good cortical density such as the glenoid rim results in failure through suture breaking or soft-tissue failure, whereas placing an ASA in a weak cortical area such as the greater tuberosity of the humeral head may result in anchor pullout.

Although ASAs showed results comparable to those of controls on load-to-failure tests, results of cyclic loading tests should be evaluated with caution. Test protocols and displacement cut-off values in those studies are inconsistent (Tables 1 and 2). Lack of a standard test protocol seems to be an important drawback for comparing anchors. Authors used differing ranges of cyclic tests varying between 100 and 500 times. ${ }^{3,4,6,7,10,11,13,14}$ As the number of cycles increases up to $5 \times$, this can significantly affect the outcomes. Cutoff values for evaluating the amount of displacement is another area of debate. Some authors used $2 \mathrm{~mm}$ as a level for significant displacement during cyclic loading, whereas others used $5 \mathrm{~mm}$. In a real-world scenario, a displacement more than $2 \mathrm{~mm}$ in a labral fixation would interfere with healing.

Few authors studied the insertion angle, the pretensioning or the drilling depth of ASA anchors. Oh et al. showed that ASAs demonstrated better mechanical properties when inserted at a more vertical angle (90 and 75 degrees) than 45 degrees. ${ }^{15}$ The stability of an ASA depends mainly on the cortical bone, so insertion of an all-suture anchor using a vertical vector seems to be logical. Dwyer et al. found that 60 Newton pretensioning an ASA ensures that the anchor is well fixed, consistently eliminating laxity and displacement. ${ }^{4}$ Because of the possibility of a handset ASA to become loose and displace prior to reaching high tension forces, pretensioning the ASA by a predetermined force seems to be useful. Ruder et al. reported less displacement by decreasing the preset drill depth of a specific ASA. ${ }^{9}$ Cortical thickness at the insertion point of an ASA seems to be the main determinant of drilling depth. As the cortical thickness increases, drill depth should be increased to ensure a proper anchor deployment. An improper drill depth may cause 2 major problems. A thick cortex associated with a short drill depth may prevent adequate suture deployment. However, longer drill depths will position the anchor far from the cortex, which may leave a small amount of spongious bone between the anchor and the near cortex. This may cause a pistoning motion and interfere with the stability of the anchor. A zone-specific and/or personalized insertion depth for ASAs may help surgeons get the most out of ASAs.

Clinical findings of patients treated with ASAs for arthroscopic shoulder instability and rotator cuff repair 
showed satisfactory results and little increase in the complication rate (retear, revision surgery because of loose anchor) (Table 4). ${ }^{18}$ These clinical study results seem correlated with the biomechanical studies. Patients treated with ASAs for the purposes of either labral or rotator cuff repair were radiologically investigated in postoperative period and showed very little bone reaction or cyst formation. These findings counter the study of Pfeiffer et al., ${ }^{6}$ who showed consistent cavity formation and significant expansion of the drill tunnel in an in vivo study in the canine glenoid. However, there are not enough clinical data to define the risks for cyst formation using the ASAs.

Current data retrieved by this review lead us to the following observations:

- The weakest link in an all-suture anchor repair construct seems to be the cortical structure; therefore, the surgical decision should be based on the bone quality.

- Various ASAs have differing application techniques and preset drill depths. Surgeons should be familiar with these anchors to get the most out of them.

- Standardized mechanical protocols to test anchors are still lacking. Any direct comparison among various mechanical studies should be made with great caution.

- A perfect experimental model for bone is still missing. Biphasic polyurethane foam seems to be the best current option.

The principal limitation of the study is that a relatively small number of studies was identified, and they used widely diverse biomechanical measurement methodologies. Second, investigations were done in various soft tissue structures, such as ligamentous (labral) or tendinous (rotator cuff and biceps tendon) tissues.

\section{Conclusions}

In conclusion, all suture anchors have similar or better mechanical properties than regular anchors. Low-profile design seems to be an important advantage that would preserve bone tissue during application. Radiologically investigated case series showed very little bone reaction or cyst formation in patients treated with ASAs. Clinical findings in these patients who underwent arthroscopic shoulder and acetabular labral repair and rotator cuff repair showed satisfactory results and had low complication rates. However, these case series cannot distinguish between the possible clinical benefits and/or risks of ASAs.

\section{References}

1. Barber FA, Herbert MA, Hapa O, et al. Biomechanical analysis of pullout strengths of rotator cuff and glenoid anchors: 2011 update. Arthroscopy 2011 1;27:895-905.
2. Mazzocca AD, Chowaniec D, Cote MP, et al. Biomechanical evaluation of classic solid and novel all-soft suture anchors for glenoid labral repair. Arthroscopy 2012;28: 642-648.

3. Barber FA, Herbert MA. Cyclic loading biomechanical analysis of the pullout strengths of rotator cuff and glenoid anchors: 2013 update. Arthroscopy 2013;29:832-844.

4. Barber FA, Herbert MA. All-Suture Anchors: Biomechanical analysis of pullout strength, displacement, and failure mode. Arthroscopy 2017;33:1113-1121.

5. Dwyer T, Willett TL, Dold AP, et al. Maximum load to failure and tensile displacement of an all-suture glenoid anchor compared with a screw-in glenoid anchor. Knee Surg Sports Traumatol Arthrosc 2016;24:357-364.

6. Pfeiffer FM, Smith MJ, Cook JL, Kuroki K. The histologic and biomechanical response of two commercially available small glenoid anchors for use in labral repairs. J Shoulder Elbow Surg 2014;23:1156-1161.

7. Douglass NP, Behn AW, Safran MR. Cyclic and load to failure properties of all-suture anchors in synthetic acetabular and glenoid cancellous bone. Arthroscopy 2017;33:977-985.

8. Erickson J, Chiarappa F, Haskel J, et al. Biomechanical comparison of a first- and a second-generation all-soft suture glenoid anchor. Orthop J Sports Med 2017;5: 2325967117717010.

9. Ruder JA, Dickinson EY, Habet N, et al. Slight reduction in the insertion depth for an all-suture anchor decreases cyclic sisplacement in the shoulder glenoid. Arthroscopy 2018;34:1384-1390.

10. Chiang FL, Hong CK, Chang CH, et al. Biomechanical comparison of all-suture anchor fixation and interference screw technique for subpectoral biceps tenodesis. Arthroscopy 2016;32:1247-1252.

11. Hong CK, Hsu KL, Kuan FC, et al. Biomechanical evaluation of a transtendinous all-suture anchor technique versus interference screw technique for suprapectoral biceps tenodesis in a cadaveric model. Arthroscopy 2018;34: 1755-1761.

12. Galland A, Airaudi S, Gravier R, et al. Pullout strength of all suture anchors in the repair of rotator cuff tears: A biomechanical study. Int Orthop 2013;37:2017-2023.

13. Goschka AM, Hafer JS, Reynolds KA, et al. Biomechanical comparison of traditional anchors to all-suture anchors in a double-row rotator cuff repair cadaver model. Clin Biomech (Bristol, Avon) 2015;30:808-813.

14. Nagra NS, Zargar N, Smith RD, Carr AJ. Mechanical properties of all-suture anchors for rotator cuff repair. Bone Joint Res 2017;6:82-89.

15. Oh JH, Jeong HJ, Yang SH, et al. Pullout strength of allsuture anchors: Effect of the insertion and traction angle: A biomechanical study. Arthroscopy 2018;34: 2784-2795.

16. Willemot L, Elfadalli R, Jaspars KC. Radiological and clinical outcome of arthroscopic labral repair with allsuture anchors. Acta Orthop Belg 2016;82:174-178.

17. Byrd JWT, Jones KS, Loring CL, et al. Acetabular allsuture anchor for labral repair: Incidence of intraoperative failure due to pullout. Arthroscopy 2018;34: 1213-1216. 
18. Van der Bracht H, Van den Langenbergh T, Pouillon M, et al. Rotator cuff repair with all-suture anchors: A midterm magnetic resonance imaging evaluation of repair integrity and cyst formation. J Shoulder Elbow Surg 2018;27:2006-2012.

19. Liberati A, Altman DG, Tetzlaff J, et al. The PRISMA statement for reporting systematic reviews and meta-analyses of studies that evaluate health care interventions: Explanation and elaboration. BMJ 2009;21: b2700.

20. Slim K, Nini E, Forestier D, et al. Methodological index for non-randomized studies (minors): development and validation of a new instrument. ANZ J Surg 2003;73: 712-716. 